\title{
Dynamic and Advanced Security for Data Storage in Distributed Environment
}

\author{
Kruthiventy Bhargavi, D. Veeraiah
}

\begin{abstract}
With the fast improvement of innovation and computer technology, cloud-based services administrations have form into an extremely rich research area. $\mathrm{CB}$ administrations give customers which accommodation, yet additionally bring different issues. In this manner, the learning of access control plan to ensure clients' confinement in cloud condition is critical. In this paper, we present an entrance control framework with advantage division dependent on security insurance. we partition the clients into individual area (PSD) and open space (PUD) sensibly. In the PSD, we put $(R / W)$ get to authorizations for clients separately. The total key encryption (KAE) is abused to execute the read access consent that improves get to effectiveness. $A$ high level of patient protection is all the while ensured by abusing a Firm dependent on improved characteristics (IABS) that can decide the clients compose get to. For PUD clients, encryption dependent on progressive properties (HABE) is connected to evade single-purpose of disappointment issues and confounded key circulation. The aftereffect of the activity and task tests demonstrates that the PS-ACS plan can accomplish security insurance in cloud-based administrations.
\end{abstract}

Index Terms: Access Control, Data sharing, Security assurance, $\mathrm{CB}$ Services.

\section{INTRODUCTION}

With the rapid advancement of cloud computing, in general huge information and open cloud administrations have been used. Clients can store their information in cloud management and rely on the specialized organization in the cloud to give access to information to different clients. Be that as it may, the cooperative of cloud specialists will never be able to be completely reliable again. Since it can give access to information to some illicit or aggressor clients, in addition to income. For customers, it is important to exploit guarantee the protection of information. In this way, the investigation of the access control plan to ensure the protection of customers in cloud conditions is of an incredible centrality. Given that the conventional access control methodology [1] cannot adequately deal with the security problems that exist in the exchange of information, different plans have been proposed to achieve the encryption and deciphering of the exchange of information.

\section{LITERATUTRE REVIEW}

In 2007, Bethencourt et al. [2] proposed for the first time the encryption based on the text approximation property (CP-ABE). Anyway, this plan does not think about the

Revised Manuscript Received on July 18, 2019.

Kruthiventy Bhargavi, M.Tech student, Dept. of Computer Science and Engineering, Lakireddy Bali Reddy College of Engineering (Autonomous), Mylavaram, Krishna Dt., 521230, Andhra Pradesh, India.

Dr D. Veeraiah, Professor, Dept. of Computer Science and Engineering, Lakireddy Bali Reddy College of Engineering (Autonomous), Mylavaram, Krishna Dt., 521230, Andhra Pradesh, India. the management of distributed storage and, in addition, to

denial of access authorizations. Attrapadung et al. [3, 4] we think of two ABE conspiracies us-revocable. In any case, they are not appropriate in the re-appropriation condition. In 2011, Hur et al. [5] presents a fine-grained waiver chart, but can, without much problem of escrow. Lewko et al. [6] used $\mathrm{ABE}$ from multiple experts (MA-ABE) to address the key problem of custody. Be that as it may, the input approach is not flexible. Then, Li et al. [7] introduced an information exchange plan dependent on the encryption of key features, which invests distinctive access authorizations to several clients. However, it lacks efficiency-cy. Xie et al. [8] introduced a revocable conspiracy of $\mathrm{CP}-\mathrm{ABE}$. In contrast, and the time plan, in the key update stage, the calculation heap of the head of information management will be significantly reduced. Liang et al. [9] proposed a consortium of CP-ABE intermediate encryption that supports any monotonic access structure. $\mathrm{Be}$ that as it may, its development that is worked in the bilinear application of compound request cannot be changed to the bilinear application of main request. In 2014, Chu et al. [10] proposed calculation of aggregate key encryption, which correctly abbreviates the length of the encrypted text and the key, however, only by the circumstance in which the owner of the information knows the character of the client. The previous plans only highlight a part of the exam, and neither do they have a demanding uniform standard. In this document, we present a progressively methodical, adaptable and efficient access control suite. For this, we assume the commitments of principle that accompany it:

1) We propose a new access control system called PSACS, which is a proportion of benefits that depends on security insurance. In order to obtain the consent of reading, in the PRD, the Code of Clustering of Key Agents (KAE) conspires, which greatly improves the effectiveness of the effectiveness, is adopted. In addition, in PUD, we built another encryption frame based on multiple creative cipher text focus (CP-ABE) features with competent deciphering to maintain a strategic distance from single-purpose problems of disappointment and entangled key transport, and we plan a repudiation of productive quality Technique for it.

2) Compared to the MAH-ABE conspiracy that does not allude to composite access control, we misused a Signature Plan based on enhanced attributes (IABS) [11-13] to authorize composite access control in the PRD. In this way, the client can pass the brand verification of the server in the cloud without revealing the character, and effectively alter the registration.

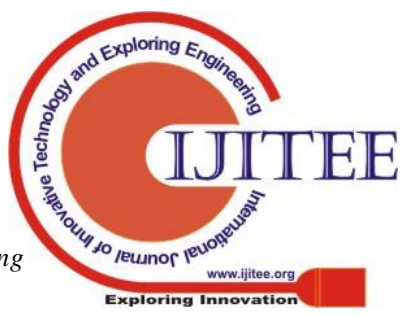


3) We perform a security and execution exam of our proposed PS-ACS chart. The results of utility and recreation give information security in the satisfactory execution of the agreement, and demonstrate the ability to carry out the plan.

\section{SYSTEM ARCHITECTURE}

The following architecture outline for the most part speaks to the progression of solicitations among clients and SaaS STORAGE, SaaS encryption module in the mists. In this situation, the general framework is structured in isolated n-tires, explicitly utilizing three layers called customer layer, SaaS cloud layer gave using java jdbc sources and java servlets for mysql and the SaaS security layer with encryption module in Java sources. The reconciliation module is accomplished utilizing java web administrations. This project was implemented using entire architecture

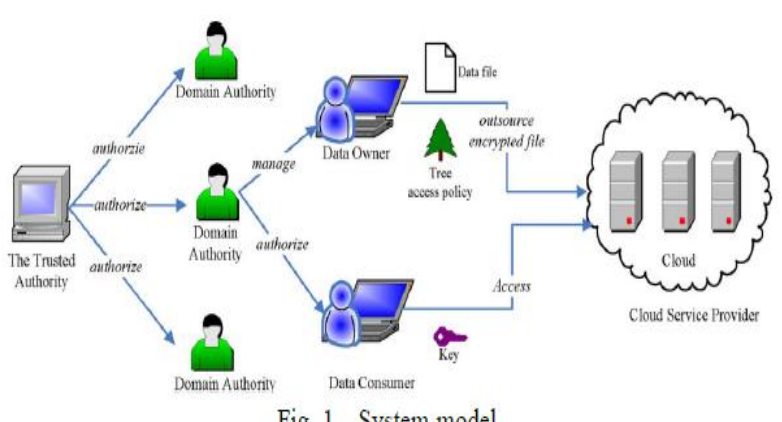

\section{PROPOSED SYSTEM}

$>$ Propose to manage the issue of executing a convention to get a trial of ownership of information in the cloud, in some cases called Solution dependent on various leveled characteristics (HASBE).

$>$ This issue endeavours to get and confirm a test that the information put away by a client in a remote information stockpiling in the cloud (called distributed storage records or essentially documents) are not adjusted by the document through hash and, for Therefore, the respectability of the information is guaranteed.

$>$ The proposed framework does not infer the age of the exceptional key, it produces various keys, which improves the protection of the clients. We encode just a couple of bits of information per information square, along these lines diminishing computational over-burden.

$>$ In our information respectability convention, the verifier must store just a single cryptographic key in the cloud, paying little mind to the span of the information document $\mathrm{F}$ and two capacities that produce an irregular arrangement.

$>$ The verifier does not store any information with it, the key stays in the vault of the cloud.

$>$ This method includes the accompanying modules.

1. Arrangement stage

- Age of metadata.
- Scramble the metadata.

- Connections of metadata.

2. Verification stage utilizing Cloud Key

The proposed framework does not infer the age of the extraordinary key, it produces different keys, which improves the protection of the clients.

The proposed framework utilizes AES and SHA for the age of different keys. It accepts properties as information sources and a few keys are produced and encoded.

\section{ACCESS CONTROL SCHEME IN PUD \& SECURITY, PERFORMANCE ANALYSIS IN PRD}

The PUD is portrayed by countless clients, countless credits having a place with the client, the executives of the multifaceted nature and inconclusive character of the clients. In perspective on the above attributes, the client can just have the perused access authorization. In spite of the fact that the property based encryption plot (CP-ABE) can accomplish access control, it cannot address the issues of a mind boggling cloud condition. In the customary CP-ABE conspire, there is just one property specialist in charge of quality administration and key circulation. The specialist can be a college enrolment office, the HR branch of the organization or legislative instructive associations, and so forth. The proprietor of the information characterizes the entrance approaches and encodes the information records as per this arrangement. Every client is circulated a key identified with its trait. For whatever length of time that the client's traits consent to the entrance strategy, he can decode the record. In any case, if there is just a single expert in the framework and all open and private keys are issued by the specialist. Two issues will show up in the down to earth application:

- In the functional condition of the cloud, there are numerous experts and every specialist in its very own field oversees some portion of the qualities of the clients. The qualities that the client has are issued by various specialists. For instance, an information proprietor might need to impart his or her restorative data to a client who has the therapeutic trait issued by the medicinal organizations and the quality of the medicinal scientist by the center staff. Appropriately, abusing different specialist is increasingly sensible in reasonable situations.

- If there is just a single expert, the whole conveyance of the keys is conveyed by a confided in power. Visit association between the client and they believed expert won't just carry bottlenecks to the framework's heap limit, yet will likewise expand potential security dangers.

\subsection{Security Analysis}

In PRD, clients can just decode the records comparing to the additional keys got and don't approach different documents, so the proprietor of the information controls the entrance authorizations of the clients. At the point when the information record is adjusted, in spite of the fact that CA is trusted, the parameters and framework repudiation

Published By:

Blue Eyes Intelligence Engineering \& Sciences Publication 
The directions are created by the CA. The mark arrangement is figured by the proprietor of the information and sent legitimately to the server in the cloud. The CA does not know the mark arrangement. Accepting that CA cannot be approved, as long as the CA characteristics cannot fulfil the entrance approach, it isn't substantial to adjust the document. In this manner, compose get to consents still have a place with the proprietor of the information. During the time spent marking the clients, the mark key is just identified with the characteristics of the clients, so the client's personality is secure. When all is said in done, the
IABS plan can secure the protection of the clients' personality.

\section{Performance analysis}

First, we created an impression for the documentation used in the presentation exam. In our KAE chart in the PRD, the framework parameters are produced by the trusting authority, which is not within our thinking. In addition, it can be determined in the frame configuration stage. What's more, the total key only needs a mixing activity, and determining a matching task is extremely fast. As shown the figure5.1a.1b comparison between existing system and proposed system

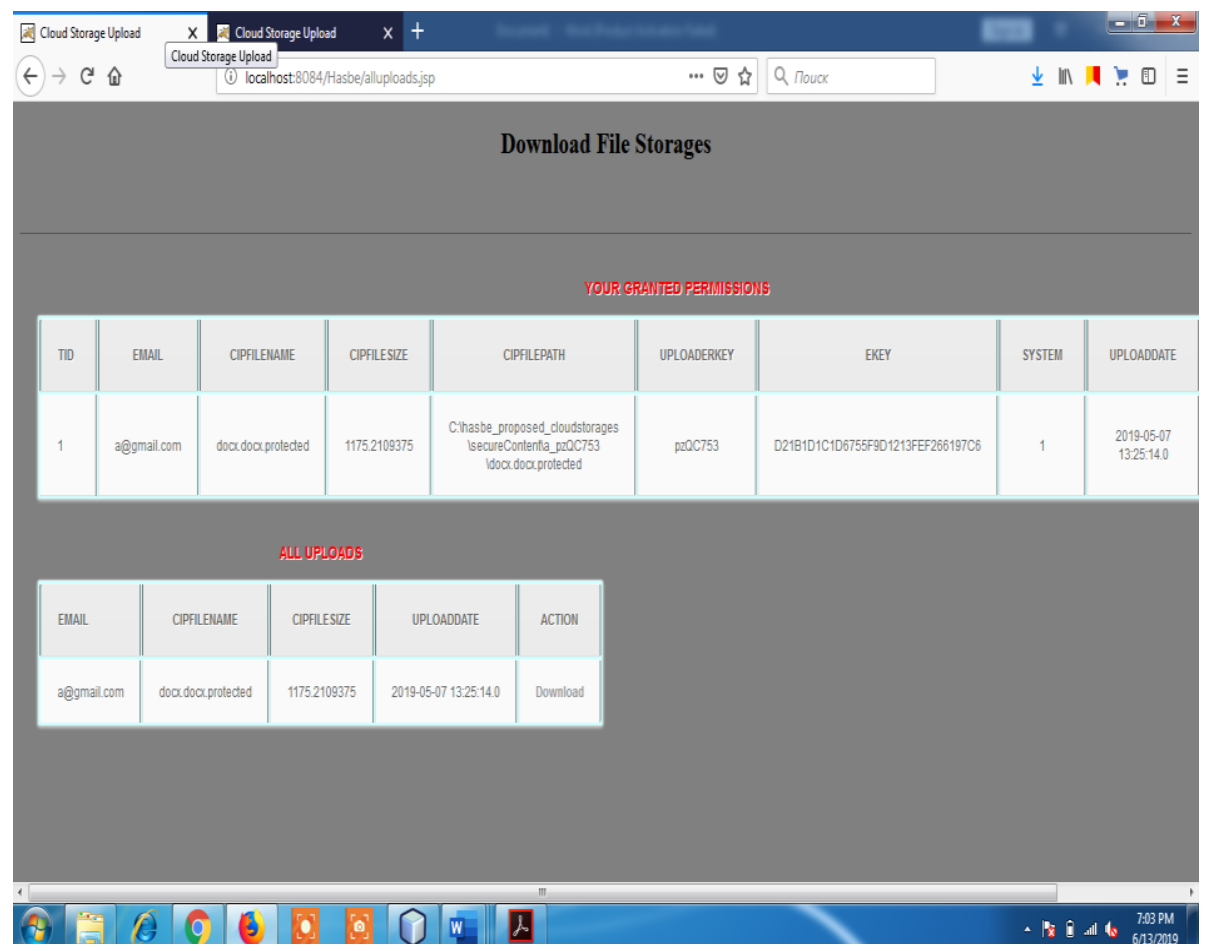

5.1a.Existing System

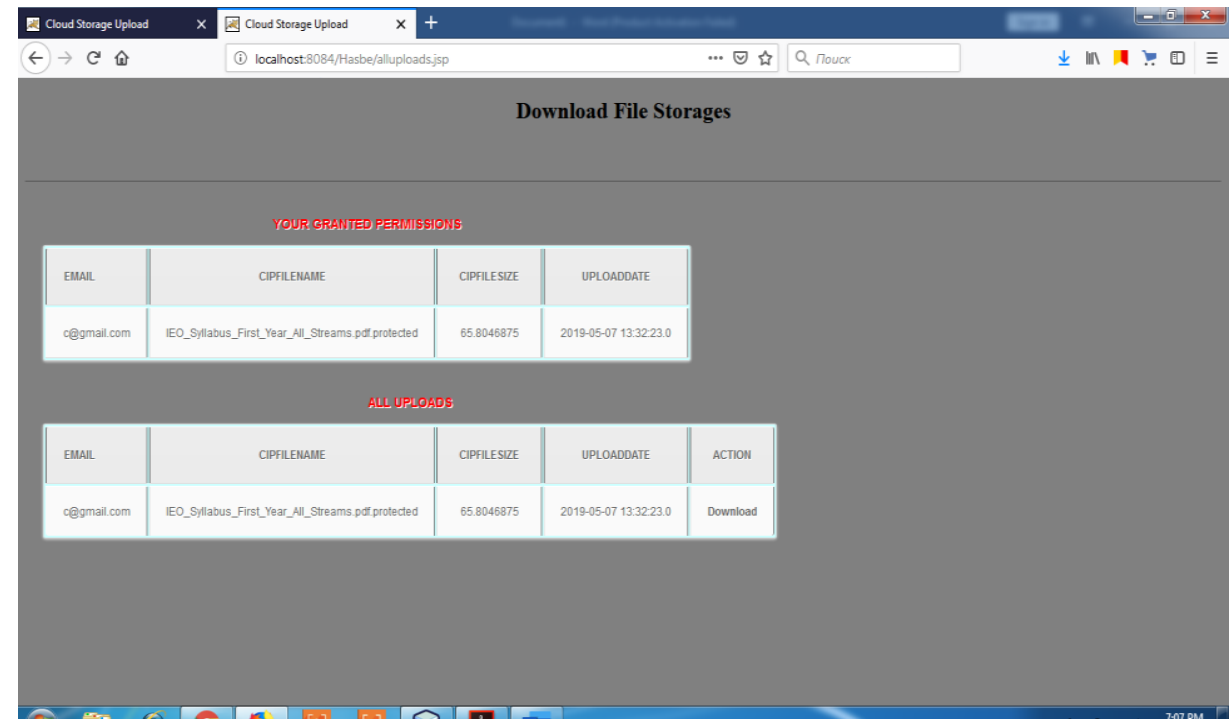

5.1b.Proposed System

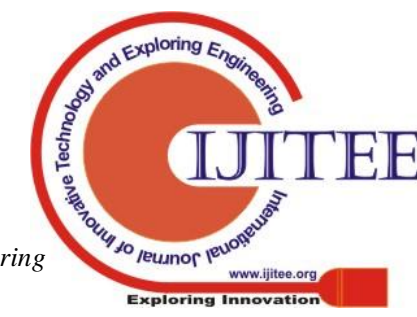




\section{RESULTS}

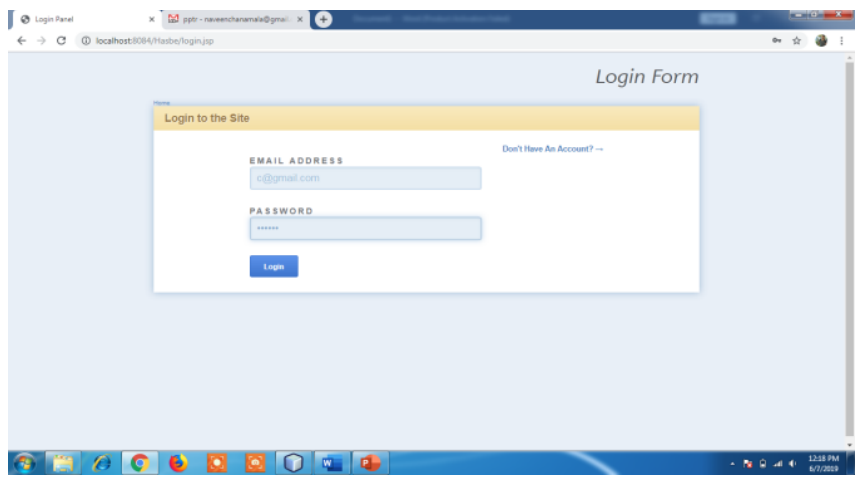

Figure 1. Login Form

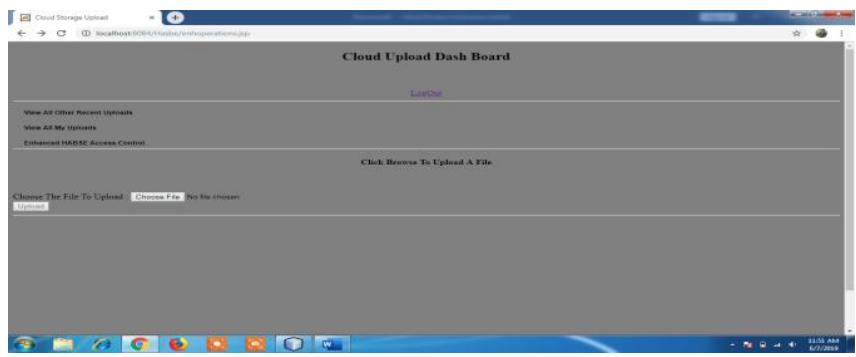

Figure 2. Upload Data
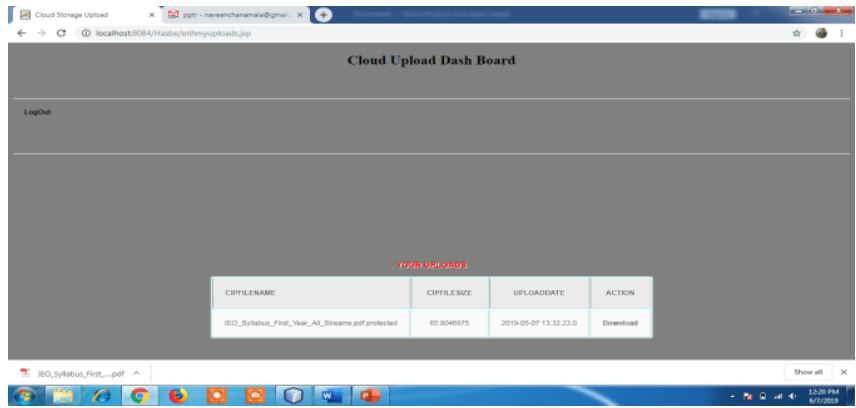

Figure 3. Cloud Upload Dash Board

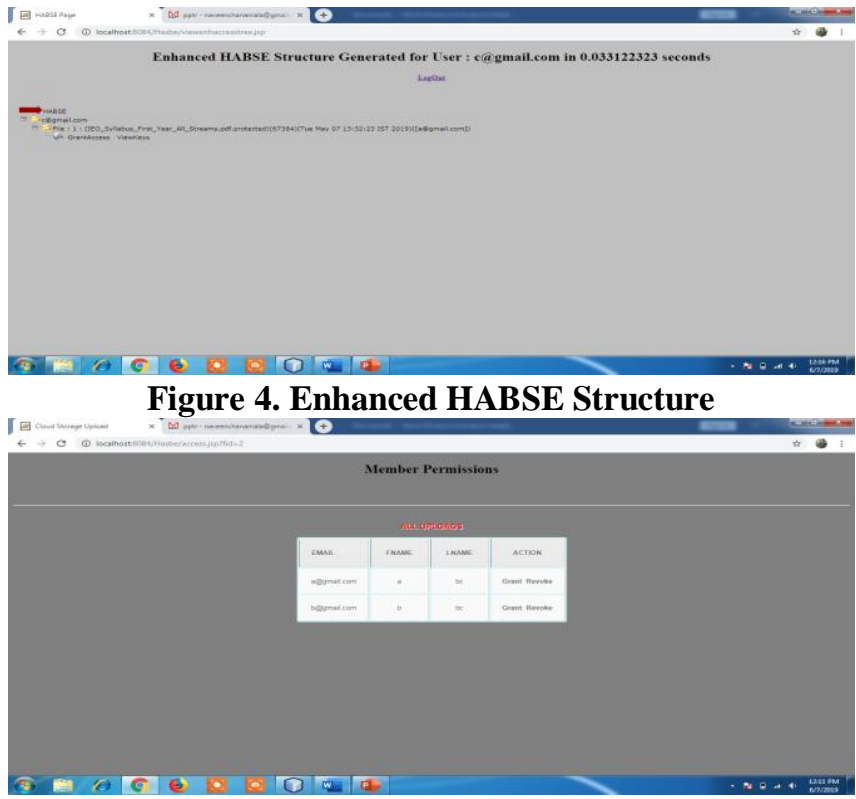

Figure 5. Member Permissions

\section{CONCLUSION}

Individual space (PRD) and open area (PUD) legitimately. In PRD, we set read and compose get to authorizations for the clients separately. To accomplish the read access authorization, the KAE plan is received, which can improve get to productivity. A high level of patient security is ensured at the same time by utilizing the IABS conspire that can decide the clients' compose get to consent. For PUD clients, we manufactured another characteristic based encryption conspire (CP-ABE) of scrambled content arrangement with different specialists with productive unscrambling to stay away from single-purpose ofdisappointment issues and confused key conveyance, and we planned an effective strategy for repudiation of properties for it. in future enhanced the advanced new privacy techniques.

\section{REFERENCES}

1. YU SH, WANG C, REN K, "Achieving Secure, Scalable, and Fine-Grained Data Access Controlin Cloud Computing", Proceedings of IEEE Conference on Information Communications 2010,pp. 1-9, 2010.

2. BETHENCOURT J, SAHAI A, WATERS B, "CiphertextPolicy Attribute-based Encryption", IEEESymposium on Security and Privacy, vol. 2008,no. 4, pp. 321-334, 2007.

3. ATTRAPADUNG N, IMAI H, "Conjunctive Broadcastand Attribute-Based Encryption", Proceedingsof Pairing-based Cryptography - Pairing2009, vol. 5671, pp. 248-265, 2009.

4. ATTRAPADUNG N, IMAI H, "Attribute-Based EncryptionSupporting Direct/Indirect RevocationModes", Proceedings of Cryptography and Coding2009, pp. 278-300, 2009.

5. HUR J, NOH D K, “Attribute-based Access Control with Efficient Revocation in Data utsourcingSystems", IEEE Transactions on Parallel andDistributed Systems, vol. 22, no. 7, pp. 1214-1221, 2011.

6. LEWKO A, WATERS B, "Decentralizing Attribute-based Encryption", Proceedings of Advancesin CryptologyUROCRYPT 2011 - 30thAnnual International Conference on the Theoryand Applications of Cryptgraphic Techniques,pp. 568-588, 2011

7. LI M, YU SH, ZHENG Y, "Scalable and SecureSharing of Personal Health Records in CloudComputing Using Attributebased Encryption",IEEE Transactions on Parallel and DistributedSystem, vol. 24, no. 1, pp. 131-143, 2013.

8. XIE X, MA H, LI J, et al, "New Ciphertext-PolicyAttributebased Access Control with EfficientRevocation", Proceedings of Information andCommunication Technology 2013, pp 373-382,2013.

9. LIANG K, MAN H A, SUSILO W, et al, "AnAdaptively CCA-Secure Ciphertext-Policy Attribute-Based Proxy ReEncryption for CloudData Sharing", Information Security Practice andExperience, pp. 448-461, 2014.

10. CHU C K, CHOW S S M, TZENG W G, "KeyAggregateCryptosystem for Scalable Data Sharingin Cloud Storage", IEEE Transactions on Paralleland Distributed Systems, vol. 25, no. 2, pp. 468-477, 2014 